\title{
The Uncertainty of Roughness Parameters
}

\author{
J. Seewig \\ Institute for Measurement and Sensor Technology, \\ Technical University of Kaiserslautern, 67663 Kaiserslautern, Gottlieb-Daimler-Straße, Germany \\ seewig@mv.uni-kl.de
}

\begin{abstract}
The process capability is an important indicator for stable and reliable manufacturing processes. Hence, the measurement uncertainty and the unambiguous calculation of geometrical parameters play an important role in quality control. A major deficiency pertains to the evaluation of the uncertainty of surface roughness parameters. Already simple roughness parameters have a large statistical variance depending on the manufacturing process and not on the measuring system. For industrial companies it is essential to find rules to express the surface roughness uncertainty, particularly for geometrical tolerancing. By describing a surface parameter as a statistical variable, it will be shown that the uncertainty can be expressed more precisely than in usual practice. As an example, the rule of thumb "six times Ra is approximately Rz" is derived from a statistical model of the surface.
\end{abstract}

Key words: uncertainty, roughness parameters, quality control, tolerancing.

\section{Introduction}

The characterization of the surface roughness differs greatly from other geometric attributes such as the shape or dimensions of a component. The surface is generally the sum of different geometric structures, the shape of which together or individually - is decisive for the function of a component. The occurring structures cover a high rescaling range. Fine submicron structures as well as micron "macro-structures" have to be evaluated.

Many different parameters are applied to reasonably characterize these complex geometric structures. Heavily used functional surfaces, usually with non-homogeneously distributed geometric structures, are particularly critical. The consequence is that the calculated parameters are widely scattered due to the surface statistics. This often leads to surface measuring devices or even manufacturing processes being incorrectly evaluated as "not capable." Furthermore, the tolerancing is often unclear.

This article offers insight into how roughness parameters dependent on the manufacturing process can be described with probability density functions. The parameters $R a$ and $R z$ are examined according to DIN EN ISO 4287 [1], and the parameters $R k, R p k$ and $R v k$ are examined according to DIN EN ISO 13565, part 2 [2].

\section{Description of roughness parameters as random variable}

The statistical spread of roughness parameters is strongly influenced by the random character of the surface properties. Thus a surface parameter acts like a random variable, the distribution of which is a factor of the surface characteristics - and the surface characteristics are determined in turn by the manufacturing process.

The following section describes the spatially discreet roughness values $r_{k}(k=1, \ldots, n$ with $n \in \mathrm{N}$ ) supplied by the measuring device as the realization of a random variable $r$ with the density function $p(r)$. To simplify the arithmetic, it is assumed that the roughness values are uncorrelated. The expectation for the random variable would be zero. This requirement is generally met by previous profile filtering.

Two known, assumed, zero-mean distribution densities will be examined to describe the roughness: a uniform distribution $u(r)$ with the range $\pm a$ and the resulting standard deviation, $\sigma_{r}=a / \sqrt{3}$ and Gaussian distribution $g(r)$ with the standard deviation $\sigma$. The uniform distribution has no direct correlation to a specific manufacturing process and is intended only to illustrate the mathematic descriptions. On the other hand, Gaussian distribution can describe manufacturing processes with indeterminate edge and symmetrical amplitude distribution. This 
includes e.g. the manufacturing process of grinding.

\section{Description of parameter $\mathrm{Ra}$ as random var- iable}

The parameter $R a$ is often used when tolerancing rough surfaces. It describes the arithmetical mean deviation of the roughness profile and is defined by

$$
R a=\frac{1}{n} \sum_{k=1}^{n}\left|r_{k}\right| .
$$

Thus the parameter $R a$ from equation (1) is the result of the averaging of the absolute values of the profile ordinates $\left|r_{k}\right|$. It can be statistically evaluated applying the deviation propagation method with the sensitivity coefficients $1 / n$. When the profile ordinates $\left|r_{k}\right|$ are regarded as the realization of the random variables $|r|$, the distribution densities $u(|r|)$ and $g(|r|)$ must be examined, which leads in turn to the expectation $\mu_{|r|}$ and the standard deviation $\sigma_{|r|}$. The correlations shown in Table 1 are obtained for the existing distribution densities (uniform distribution, Gaussian distribution) by calculating the first and second statistical moments.

Table 1: Expectation and standard uncertainty of arithmetical mean deviation of the roughness profile.

\begin{tabular}{|c|c|c|c|c|}
\hline & $\mu_{|r|}$ & $\sigma_{|r|}$ & $\mu_{R a}$ & $\sigma_{R a}$ \\
\hline$u(r)$ & $\frac{a}{2}$ & $\frac{a}{2 \sqrt{3}}$ & $\frac{a}{2}$ & $\frac{1}{\sqrt{n}} \frac{a}{2 \sqrt{3}}$ \\
\hline$g(r)$ & $\sqrt{\frac{2}{\pi}} \sigma$ & $\sqrt{1-\frac{2}{\pi}} \sigma$ & $\sqrt{\frac{2}{\pi}} \sigma$ & $\frac{1}{\sqrt{n}} \sqrt{1-\frac{2}{\pi}} \sigma$ \\
\hline
\end{tabular}

As the table shows, the overall standard deviation is reduced by the factor $1 / \sqrt{n}$ as compared to the single standard deviation. Building the sum in equation (1) has an averaging effect. This is also referred to as an "integral parameter" that provides statistically stable values. However, the disadvantage of an integral parameter is that locally pronounced structure variations that are relevant to the function (e.g. grooves or peaks) are also averaged, and the resulting parameter offers virtually no conclusion regarding the local surface texture. The parameter $R a$ is comparable to the square mean deviation of the roughness profile $R q$. While $R q$ is applied to estimate the standard deviation in the sense of the smallest square deviations (L2 norm), $R a$ can be interpreted as a robust estimation of the standard deviation based on the smallest absolute deviations (L1 norm).

\section{Description of parameters Rt and Rz as ran- dom variable}

The parameter $R t$ describes the roughness depth, meaning the difference between the profile peak and the profile valley within a specified measuring length. It is defined as:

$$
R t=\max _{k=1 . . n}\left(r_{k}\right)-\min _{k=1 . . .}\left(r_{k}\right)
$$

$R t$ reacts very sensitively to outliers and is used particularly to detect cracks in the boundary layer. Examination of the uncertainty for $R t$ can no longer be realized with the simple deviation propagation method, as it could be in the case of the parameter $R a$. The probability density $p(r)$ used as the basis for all profile ordinates $r_{k}$ must be examined here.

The first step is to determine the probability $\Delta P(X)$ that a profile value will assume exactly the value $X=\min \left(r_{k}\right), k=1, . ., n$, and that the remaining $n-1$ profile values are within the interval $[X, X+R t]$. Since there are $n$ possible profile points, it follows that:

$\Delta P(X)=n\left(\int_{X}^{X+R t} p(\xi) d \xi\right)^{n-1}$.

The result of equation (3) for the probability density $p(R t, n)$, when all possible variation for $X$ are considered, is:

$$
p(R t, n)=\frac{\partial}{\partial R t} \int_{-\infty}^{\infty} n\left(\int_{X}^{X+R t} p(\xi) d \xi\right)^{n-1} p(X) d X
$$

For instance, example (4) for uniform distribution becomes much simpler:

$$
\begin{aligned}
& p(R t, n)=\frac{\partial}{\partial R t} \int_{-a}^{a-R t} n\left(\int_{X}^{X+R t} \frac{1}{2 a} d r\right)^{n-1} \frac{1}{2 a} d X \\
& =n(n-1)\left(\frac{R t}{2 a}\right)^{(n-2)}\left(1-\frac{R t}{2 a}\right) \frac{1}{2 a}, \quad 0 \leq R t \leq 2 a
\end{aligned}
$$

Equation (5) can be used to find analytic solutions for the expectation and for the standard deviation of the random variable $R t$. These expressions are shown in Table 2.

Table 2: Expectation and standard uncertainty of the roughness depth for uniform distribution.

\begin{tabular}{|c|c|c|c|}
\hline$\mu_{r}$ & $\sigma_{r}$ & $\mu_{R t}$ & $\sigma_{R t}$ \\
\hline 0 & $\frac{a}{\sqrt{3}}$ & $2 a \frac{(n-1)}{(n+1)}$ & $2 a \sqrt{\frac{2(n-1)}{(n+2)(n+1)(n+1)}}$ \\
\hline
\end{tabular}

In the case of Gaussian distribution, equation (4) is analytically no longer a closed form ex- 
pression, meaning that numerical integration should be performed. Figure 1 shows the progression $\mu_{R t} / \mu_{R a}$ and figure 2 the progression
$\sigma_{R t} / \sigma_{r}$ along the course of roughness values $n$, for uniform distribution as well as for Gaussian distribution

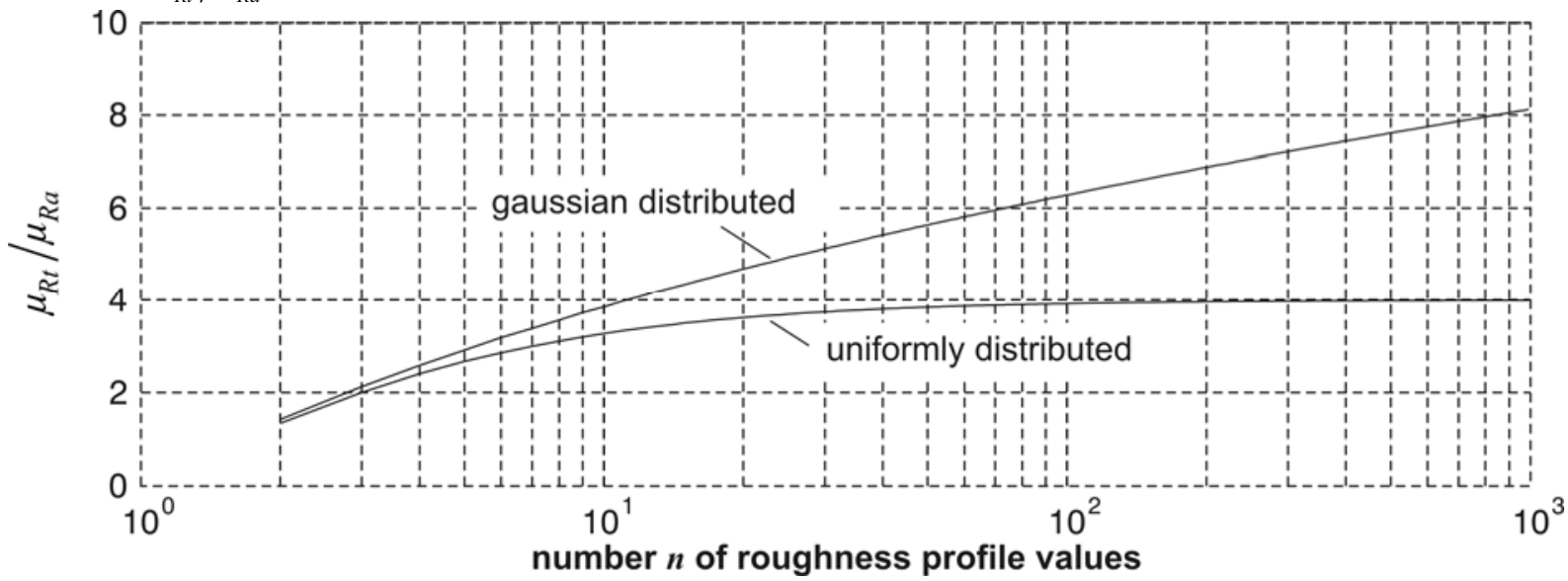

Fig. 1. Quotient $\mu_{R t} / \mu_{R a}$ of expectations as a factor of the number $n$ of roughness values.

As figure 1 shows, the quotient $\mu_{R t} / \mu_{R a}$ of uniform distribution converges towards the value 4 . This behavior is plausible because uniform distribution has upper and lower limits and its expectation for $R t$ has to assume a maximum as the quantity of profile ordinates increases. Thus the quotient $\mu_{R t} / \mu_{R a}$ for Gaussian distri- bution increases very monotonically, since the probability that individual outliers will be evaluated becomes greater as the quantity of profile ordinates increases. When $n=1000$ values, the quotient for $\mu_{R t} / \mu_{R a}$ is about 8 . This value is often reached in practical applications.

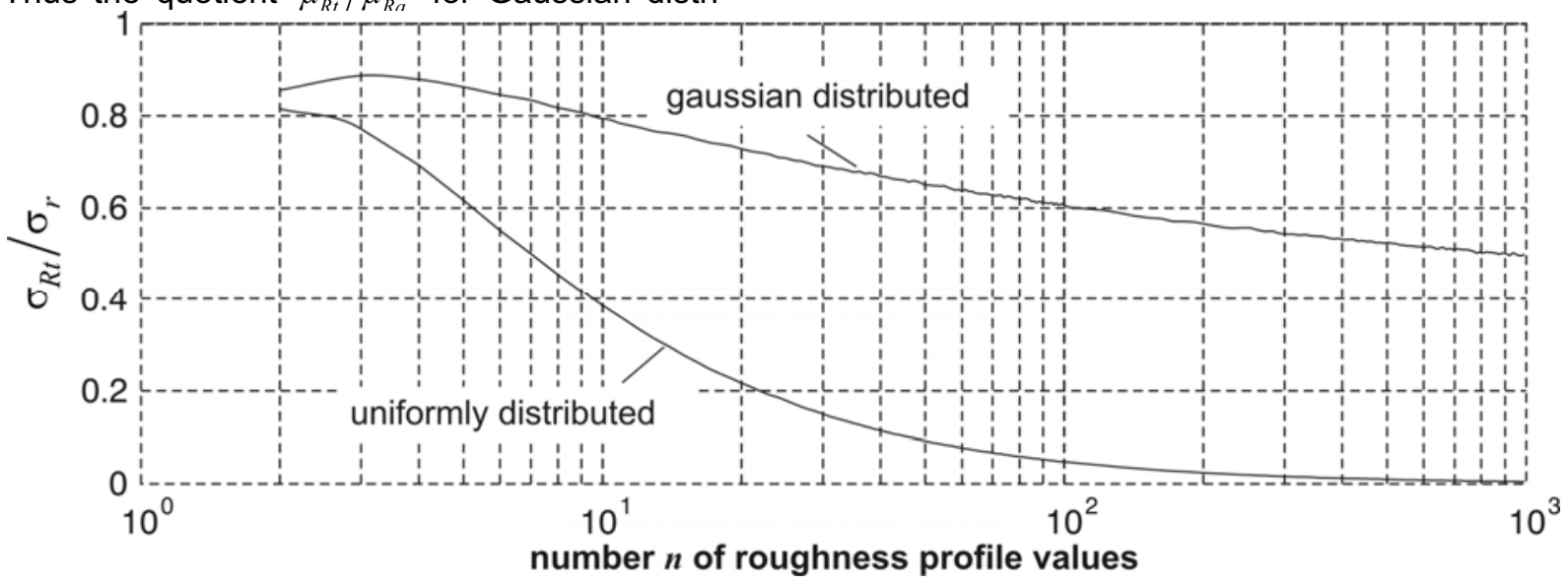

Fig. 2. Quotient $\sigma_{R t} / \sigma_{r}$ of standard deviations as a factor of the number $n$ of roughness values.

The quotient $\sigma_{R t} / \sigma_{r}$ for uniform distribution (Refer to Figure 2) behaves proportionally to $1 / \sqrt{n}$. This behavior is plausible too, because, as described earlier, the expectation converges towards a finite value. In contrast, the course of standard deviation of $R t$ for Gaussian distribution as a factor of the quantity of roughness values proceeds very differently. With 1000 values, $\sigma_{R t}$ is reduced to only half of the value $\sigma_{r}$ of the single ordinates. So the probability density function of the roughness ordinates has a major effect on the standard deviation of the parameter $R t$.

The standard deviation for the parameter $R z$ can be stated applying the estimations for the parameter $R t . R z$ is the mean value of five $R t$ values, each of which is determined within a single sampling length. When $n$ described the quantity of roughness values for each single sampling length, the standard deviation is reduced to $\sigma_{R z}=\sigma_{R t} / \sqrt{5}$.

\section{Expectations for the parameters Rk, Rpk and Rvk}

The basis for the parameters according to DIN EN ISO 13565, part 2 is the Abbott curve, which is defined as an inverse amplitude distribution function of the roughness ordinates and describes the material ratio $\mathrm{Mr}$ as a factor of the depth in the roughness profile (Figure 3 shows an example of the Abbott curve for Gaussian distribution of roughness ordinates). 


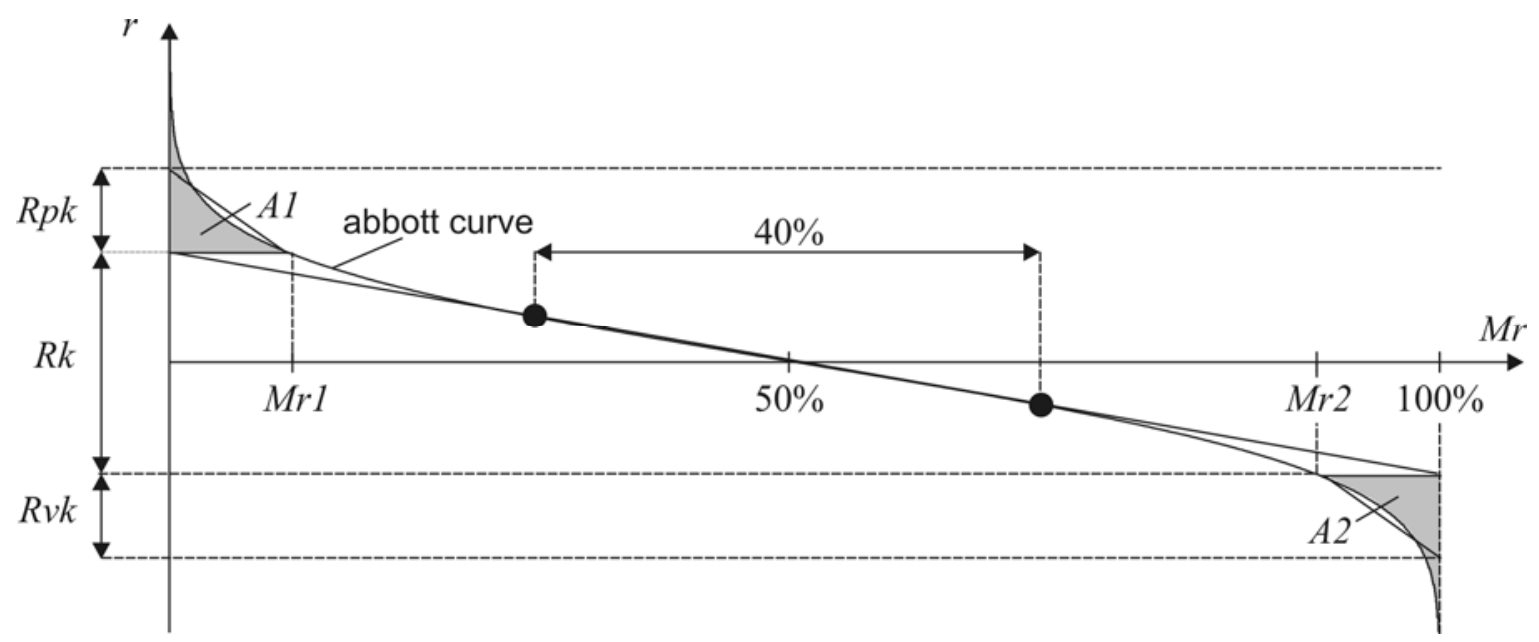

Fig. 3. Parameters $R k, R p k, R v k$ and $M r 1, M r 2$ of the Abbott curve.

To calculate the parameters, a line of best fit is calculated according to the standard, with a minimum slope for the central range of the material ratio curve that includes $40 \%$ of all of the measured profile points. The vertical spacing of the intersection of these lines at $\mathrm{Mr}=0 \%$ and $M r=100 \%$ is referred to as the core roughness depth $R k$. The intersections of the lines with the Abbott curve produce the material ratios $M r 1$ and $M r 2$. The parameters $R p k$ and $R v k$ are each designated as the side length of a triangle with an area equal to the peak area $A 1$ or the groove area $A 2$.

The Abbott curve is efficiently numerically calculated by sorting the profile ordinates in descending order. Each profile ordinate $\tilde{r}_{k}$ $(k=1, \ldots, n$ mit $n \in \mathrm{N})$ with $\tilde{r}_{1} \geq \tilde{r}_{2} \geq . . \geq \tilde{r}_{n}$ corresponds to a material increase of $100 / n$ percent. $\tilde{r}_{k}$ can be statistically described by the probability density function

$$
\begin{aligned}
& \tilde{p}_{k}(r)=\frac{n !}{(n-k) !(k-1) !} \\
& \frac{\partial}{\partial r} \int_{-\infty}^{r}\left(\int_{-\infty}^{X} p(\xi) d \xi\right)^{n-k}\left(\int_{X}^{\infty} p(\xi) d \xi\right)^{k-1} p(X) d X
\end{aligned}
$$

Equation (6) can be simply derived having in mind that for each position $k$, there are $n-k$ values which are lower than $X$ and $k-1$ values which are greater than $X$.

Now the case is to be examined in which there is an infinite number of roughness ordinates and the Abbott curve follows a theoretically ideal course. Assuming that the roughness ordinates have no mean values and Gaussian distribution, the following distribution function results:

$$
\operatorname{Mr}(r)=\int_{r}^{\infty} g(\xi) d \xi=\frac{1-\operatorname{erf}\left(\frac{r}{\sqrt{2} \sigma}\right)}{2}
$$

with the so-called "error function"

$$
\operatorname{erf}(r)=\frac{2}{\sqrt{\pi}} \int_{0}^{r} e^{-\xi^{2}} d \xi
$$

The Abbot curve shown in figure 3 is calculated from the inverses of the distribution function according to equation (7):

$$
r(M r)=\operatorname{erf}^{-1}(1-2 M r) \sqrt{2} \sigma
$$

To calculate the parameter $R k$, a straight line of best fit in the range of $30 \%-70 \%$ (corresponds to cover of $40 \%$ ) of the material ratio is adapted for symmetry reasons. Thus the course of the function of the straight line is $r(M r)=m(M r-0.5)$, whereby $m$ is the slope of the straight line. The slope is calculated according the standard by minimizing the least squares sum between the Abbott curve and the straight line, here in the range $0.3 \leq M r \leq 0.7$ :

$$
\min _{m}\left(\int_{0.3}^{0.7}\left(\operatorname{erf}^{-1}(1-2 M r) \sqrt{2} \sigma-m(M r-0.5)\right)^{2} d M r\right)
$$

Equating the first partial derivative after the unknown slope to zero leads to the solution for the sought slope:

$$
\begin{aligned}
m & =\frac{\int_{0.3}^{0.7} \operatorname{erf}^{-1}(1-2 M r) \sqrt{2} \sigma(M r-0.5) d M r}{\int_{0.3}^{0.7}(M r-0.5)^{2} d M r} \\
& \simeq-2.5739 \sigma
\end{aligned}
$$


Applying this equation, the parameter $R k$ is calculated as

$R k=-m \simeq 2.5739 \sigma$

Taking into consideration the equation (7), the parameter $\mathrm{Mr} 1$ is calculated as

$M r 1=M r\left(\frac{R k}{2}\right)=\frac{1-\operatorname{erf}\left(-\frac{m}{\sigma 2 \sqrt{2}}\right)}{2}$

$$
\simeq 0.0991
$$

and, for reasons of symmetry, the parameter $M r 2$ is

$$
\begin{aligned}
M r 2 & =1-M r 1 \\
& \simeq 0.9009
\end{aligned}
$$

The area below the Abbott curve in the range $R k / 2<r<\infty$ is

$$
\begin{aligned}
A 1 & =\int_{R k / 2}^{\infty} \frac{1-\operatorname{erf}\left(\frac{\xi}{\sqrt{2} \sigma}\right)}{2} d \xi \\
& =\sigma\left(\frac{\exp \left(-\frac{1}{8} \frac{m^{2}}{\sigma^{2}}\right)}{\sqrt{2 \pi}}+\frac{m}{4 \sigma}\left(1-\operatorname{erf}\left(-\frac{m}{\sigma 2 \sqrt{2}}\right)\right)\right.
\end{aligned}
$$

$$
\begin{aligned}
A 1 & =\frac{R p k \cdot M r 1}{2} \\
& =R p k \cdot \frac{1-\operatorname{erf}\left(-\frac{m}{\sigma 2 \sqrt{2}}\right)}{4}
\end{aligned}
$$

Using the result from equation (13), the parameter $R p k$ is calculated as a factor of $R k$ to be

$$
\begin{aligned}
R p k & =R k\left(\frac{2 \sqrt{2} \exp \left(-\frac{1}{8} \frac{m^{2}}{\sigma^{2}}\right)}{\sqrt{\pi}\left(-\frac{m}{\sigma}\right)\left(1-\operatorname{erf}\left(-\frac{m}{\sigma 2 \sqrt{2}}\right)\right)}-1\right) \\
& \simeq R k 0.3672
\end{aligned}
$$

And for reasons of symmetry, this also applies: $R v k=R p k$.

The estimation previously performed is to be checked using an example of an actual measurement. This was done by measuring a roughness standard with a mean roughness depth of $R z=1.5 \mu \mathrm{m}$. Figure 4 shows the profile of the roughness standard along an evaluation length of $4 \mathrm{~mm}$. The amplitude distribution of the roughness standard can be described well with a Gaussian distribution. The surface topography was created by the manufacturing process of grinding.

And for the triangle of equal area, the following applies:

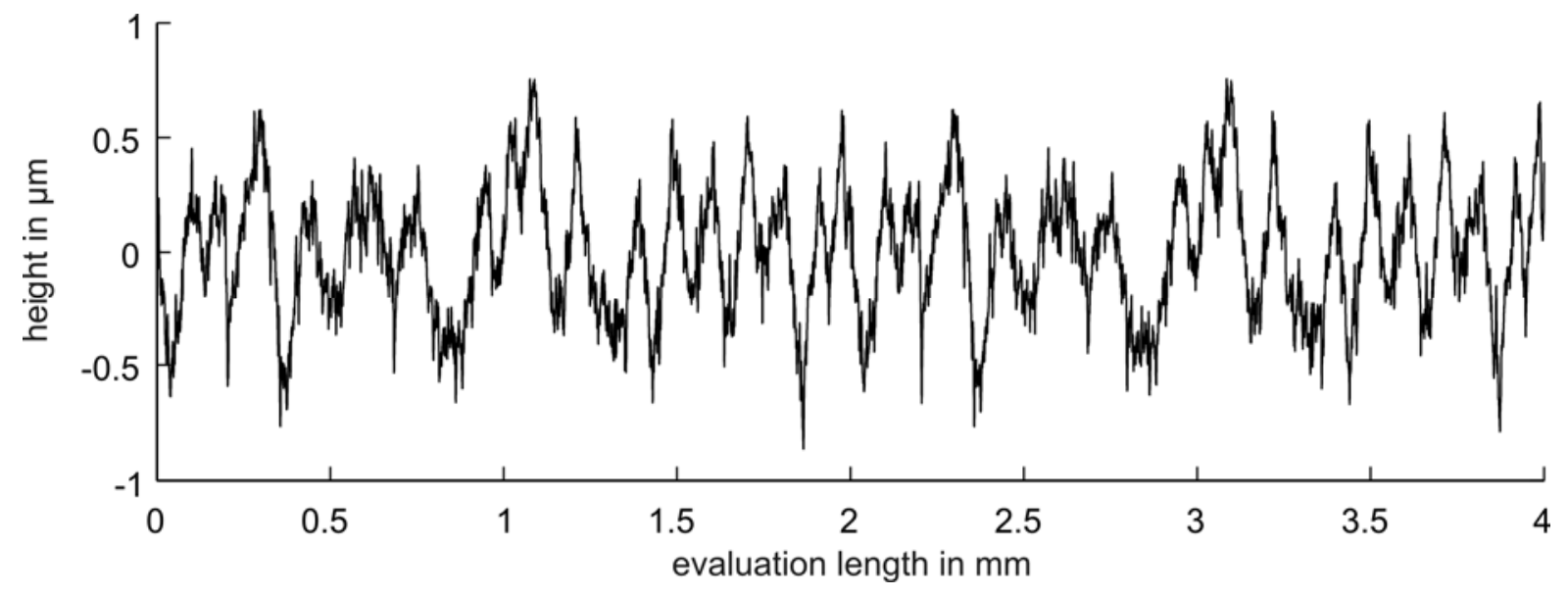

Fig. 4. Roughness profile of a standard.

The standard deviation of the profile ordinates can be estimated by the square mean deviation $\sigma \simeq R q$. Thus: $R q=281 \mathrm{~nm}$. The core roughness depth is calculated according to the norm $R k=734 \mathrm{~nm} . \quad R k \simeq R q \cdot 2.5739=723 \mathrm{~nm}$ applies to the estimation. Calculating the parameters $R p k, R v k, M r 1$ and $M r 2$ according to the standard leads to $R p k=247 \mathrm{~nm}, R v k=224 \mathrm{~nm}$, $M r 1=8.67 \%$ and $M r 2=87.95 \%$. The results of estimation from the standard deviation are $R p k=R v k=R k 0.3672=265 \mathrm{~nm}$ as well as $M r 1=9.91 \%$ and $M r 2=90.09 \%$. The calculated parameters come very close to the forecasts.

Under actual conditions, there is a finite number of profile ordinates. Sorting the profile ordinates generally leads to high uncertainty around the 
edges of the Abbott curve. The result is that particularly the paramteters $R p k$ and $R v k$ can scatter substantially. If a variance $\sigma_{k}^{2}$ and covariance $\sigma_{k, j}^{2}$ is assigned to each discrete point on the Abbott curve, the standard deviation of the parameters $R p k$ and $R v k$ is:

$$
\sigma_{R p k}=\frac{2}{M r 1 \cdot n} \sqrt{\sum_{k=1}^{\lfloor\cdot M r r\rfloor} \sigma_{k}^{2}+2 \sum_{k=1}^{\lfloor n \cdot M r 1\rfloor-1\left\lfloor\cdot \sum_{j=k+1}^{\lfloor\cdot M r 1\rfloor}\right.} \sigma_{k, j}^{2}}
$$

and

$$
\sigma_{R v k}=\frac{2}{1-M r 2 \cdot n} \sqrt{\sum_{k=[n \cdot M r 2\rceil}^{n} \sigma_{k}^{2}+2 \sum_{k=[n \cdot M r 2\rceil}^{n-1} \sum_{j=k+1}^{n} \sigma_{k, j}^{2}} .
$$

\section{Conclusion}

Surface topography seems like a random process that depends on the manufacturing method and has the effect of an input parameter on determination of parameters. This means that the parameter itself is a "natural" or "unavoidable" random variable - the statistical properties of which depend on the manufacturing process - that needs to be taken into consideration for tolerancing of a component. In contrast, the uncertainty aspects of the measuring device play only a secondary role.

\section{References}

[1] ISO 4287 (2000) Geometrical product specification (GPS) - Surface texture: Profile method Terms, definitions and surface texture parameters. International Organization of Standardization

[2] ISO 13565-2:1996, Geometrical Product Specifications (GPS) - Surface texture: Profile method; Surfaces having stratified functional properties Part 2: Height characterization using the linear material ratio curve 